\title{
PERCEPÇÃO DE JUSTIÇA PROCESSUAL NA AVALIAÇÃO DE DESEMPENHO: UMA ANÁLISE À LUZ DA INCERTEZA E DA TOLERÂNCIA À AMBIGUIDADE
}

\author{
Ilse Maria Beuren ${ }^{1}$ \\ Fernanda Kreuzberg ${ }^{2}$ \\ Leandro Franz ${ }^{3}$
}

Resumo: O estudo objetiva verificar a relação entre as medidas de avaliação de desempenho e a percepção de justiça processual à luz da incerteza e da tolerância à ambiguidade. Pesquisa descritiva com abordagem quantitativa foi realizada por meio de levantamento junto a 119 empregados de uma cooperativa de produção da região Sul do Brasil. Utilizou-se o questionário do estudo de Hartmann e Slapnicar (2012), composto de três medidas que influenciam na avaliação de desempenho e nas percepções de justiça (formalidade, voz, medidas de resultado). Para análise dos dados aplicou-se modelagem de equações estruturais. Os resultados mostram relação positiva entre formalidade e justiça processual, sendo maior em ambientes de alta incerteza de tarefas e de baixa tolerância à ambiguidade. A relação entre voz dos empregados e percepção de justiça processual é melhor explicada em ambientes de baixa incerteza e alta tolerância à ambiguidade. A relação das medidas de resultado com justiça processual apresenta-se maior para a amostra em ambientes de baixa incerteza e alta tolerância à ambiguidade. Conclui-se que formalidade, voz e medidas de resultado afetam a percepção de justiça processual dos empregados da cooperativa de produção pesquisada. Todavia, diferenças em relação ao estudo de Hartmann e Slapnicar (2012), bem como as diferenças entre as amostras relativas a incerteza de tarefas e a tolerância à ambiguidade instigam novas pesquisas.

Palavras-chave: Incerteza de tarefas. Tolerância à ambiguidade. Justiça Processual. Avaliação de desempenho.

E-mail: ilse.beuren@gmail.com - Universidade Federal de Santa Catarina

${ }^{2}$ E-mail: fernandakreuzberg@gmail.com - Universidade Federal de Santa Catarina

33-mail: franz@cooperauriverde.com.br - Faculdade La Salle de Lucas do Rio Verde 


\section{PERCEPTION OF PROCEDURAL FAIRNESS IN PERFORMANCE EVALUATION: AN ANALYSIS IN THE LIGHT OF UNCERTAINTY AND TOLERANCE TO AMBIGUITY}

Abstract: The goal of this study is to investigate the relationship between performance evaluation measures and the perception of procedural fairness in light of uncertainty and tolerance to ambiguity. Descriptive research with quantitative approach was conducted through survey to 119 employees of a production cooperative in southern Brazil. It was used a study questionnaire of Hartmann and Slapnicar (2012), composed of three measures that influence on performance evaluation and perceptions of fairness (formality, voice, result measures). For data analysis was applied structural equations modeling. The results show a positive relationship between formality and procedural fairness, being higher in environments of high uncertainty of tasks and low tolerance to ambiguity. The relationship between employee voice and perception of procedural fairness is best explained in low uncertainty environments and high tolerance to ambiguity. The relationship of result measures with procedural fairness is higher for the sample in low uncertainty environments and high tolerance to ambiguity. It is concluded that formality, voice and result measures affect the perception of procedural fairness of the employees of production cooperative surveyed. However, differences in relation to the study of Hartmann and Slapnicar (2012), as well the differences between the samples regarding tasks uncertainty and tolerance to ambiguity instigate new research.

Keywords: Uncertainty of tasks. Tolerance to ambiguity. Procedural Fairness. Performance evaluation. 


\section{INTRODUÇÃO}

O enfoque das percepções de justiça organizacional tem recebido atenção destacada por parte de estudiosos. Segundo Vand Den Bos e Lind (2002), as pessoas passam a refletir sobre o que seria considerado um procedimento justo a partir da presença de incertezas. Dessa forma concebe-se a justiça organizacional, que abrange a discussão das percepções de justiça sob a perspectiva dos empregados em relação a organização (Diekmann, Barsness, \& Sondak, 2004). No entanto, a justiça organizacional abrange diversas dimensões, sendo uma delas a justiça processual, que será abordada neste estudo.

A justiça processual conjectura-se nos procedimentos da avaliação de desempenho. Segundo Santos (2010, p. 42), o objeto de estudo da justiça processual compreende "os meios utilizados para a consecução dos fins alcançados". De acordo com a Teoria da Gestão da Incerteza, o efeito da justiça nos procedimentos de avaliação de desempenho é condicional à quantidade de tarefas incertas a que os gestores estão submetidos (Cremer, \& Sedikides, 2005; Hartmann, \& Slapnicar, 2012).

As organizações necessitam adotar medidas para avaliar o desempenho de seus empregados, considerando que estes se deparam com as incertezas das decisões dos gestores e a ambiguidade ocasionada pela falta de informações completas para que os resultados esperados sejam alcançados (Hartmann, \& Slapnicar, 2012). Complexidades desta natureza permeiam os efeitos da justiça na avaliação de desempenho.

Referente à incerteza, Vand Den Bos e Lind (2002) aduzem que pela presença de incertezas quanto a confiabilidade dos superiores, faz-se maior uso da percepção de justiça. No entanto, quando o superior exprime variabilidade no quesito confiança, os subordinados reduzem o efeito da justiça. Desse modo, quando há um deslocamento da incerteza para a certeza, diminui-se a utilização da justiça. Em relação a tolerância à ambiguidade, uma pessoa passa a se sentir desconfortável a partir do momento em que precisa confrontar informações incompletas (Hartmann, \& Slapnicar, 2012).

Lau e Buckland (2001) realizaram um estudo utilizando métricas financeiras na avaliação de resultados, que ocasionaram percepções de justiça mais elevadas. Porém, na pesquisa de Lau e Moser (2008), os resultados foram contrários, pois as métricas não financeiras apresentaram maior reflexo na justiça, como o caso da variável voz. Diante destes resultados contraditórios percebe-se a necessidade de maiores reflexões e pesquisas que analisem os efeitos da percepção de justiça processual nos procedimentos de avaliação de desempenho.

Diante dos resultados contraditórios apontados, este estudo busca contribuir para o avanço teórico e empírico desta discussão, mediante a análise da justiça processual e sua influência na formalidade (Lau, \& Buckland, 2001; Diekmann, Barsness, \& Sondak, 2004; Hartmann, 2005; Moers, 2005; Hartmann, \& Slapnicar, 2009; 2012), a voz (Korsgaard, \& Roberson, 1995; Assmar, Van Den Bos, \& Lind, 2002; Ferreira, \& Souto, 2005; Hartmann, \& Slapnicar, 2012) e nas medidas de resultado (Lau, \& Buckland, 2001; Hartmann, 2005) no processo de avaliação de desempenho.

Com base na Teoria da Gestão da Incerteza, argumenta-se que os efeitos da justiça na avaliação do desempenho podem não ocorrer de forma direta, mas por influência da quantidade de tarefas incertas que são atribuídas aos gestores, assim como a sua tolerância à ambiguidade (Hartmann, \& Slapnicar, 
2009). Destaca-se que a incerteza de tarefas refere-se a complexidade das tarefas a serem executadas pelos gestores enquanto que a tolerância à ambiguidade refere-se a necessidade de informações completas para a tomada de decisões em situações de incerteza (Hartmann, 2000; Hartmann, 2005).

Neste sentido, destaca-se outra contribuição da presente pesquisa, visto que a análise dos efeitos da incerteza de tarefas e da tolerância à ambiguidade dos subordinados vem adquirindo espaço no âmbito acadêmico, conforme é possível verificar nas pesquisas de Cremer e Sedikides (2005), Hartmann (2005), Hartmann e Slapnicar (2009; 2012).

Com vistas na lacuna percebida e nas contribuições vislumbradas com o estudo, busca-se responder a seguinte questão de pesquisa: Qual a relação entre as medidas de avaliação de desempenho e a percepção de justiça processual sob o efeito de incerteza e da tolerância à ambiguidade? Assim, o estudo objetiva verificar a relação entre as medidas de avaliação de desempenho e a percepção de justiça processual à luz da incerteza e da tolerância à ambiguidade.

O campo da pesquisa focaliza uma cooperativa de produção, considerando que, embora as cooperativas não tenham por objetivo o lucro, elas buscam agregar valor ao produto do cooperado e, consequentemente, gerar sobras a serem distribuídas aos cotistas. Portanto, as cooperativas necessitam ser tão competitivas quanto as empresas que buscam maximizar o lucro, o que exige responsabilidade com esse princípio no processo de tomada de decisão (Campos, \& Gozer, 2008).

O exposto justifica a relevância e contribuição desta pesquisa, especialmente por analisar diversos fatores que influenciam na percepção de justiça processual dos empregados (formalidade, voz e medidas de resultado). A pesquisa difere do estudo de referência, de Hartmann e Slapnicar (2012), pelo ambiente de aplicação e pela inter-relação da justiça processual e da avaliação de desempenho.

\section{REFERENCIAL TEÓRICO}

\subsection{GESTÃO DA INCERTEZA E JUSTIÇA PROCESSUAL}

A Teoria da Gestão da Incerteza está relacionada com a justiça organizacional ao propor às pessoas a necessidade de gerir as incertezas inerentes ao contexto das organizações (Diekmann, Barsness, \& Sondak, 2004; Hartmann, \& Slapnicar, 2012). Van Den Bos e Lind (2002) e Thau, Bennett, Mitchell e Marrs (2009) advertem que a incerteza é necessária no âmbito da previsibilidade e da redução da incerteza. Nesse sentido, Thau et al. (2009) destacam que um dos maiores desafios das pessoas é justamente lidar com as incertezas, principalmente das relações sociais.

Hartmann e Slapnicar (2012) argumentam que pela Teoria da Gestão da Incerteza, considera-se que a incerteza é o principal fator moderador para a percepção de justiça, afetando o entendimento do que seria considerado justo pelas pessoas. Dessa forma, assume-se a necessidade de justiça organizacional para o gerenciamento das incertezas enfrentadas na organização. A incerteza faz com que as pessoas se preocupem com o controle da mesma, refletindo e interferindo na qualidade de vida e dos resultados que podem vir a acontecer, visto que afeta as cognições, percepções, sentimentos e o comportamento das pessoas (Van Den Bos, \& Lind, 2002; Thau et al., 2009).

A incerteza ameaça o senso geral das pessoas, por isso buscam-se formas de amenizá-la no contexto interno das empresas, considerando que os empregados lidam com a incerteza e tentam encontrar informações mais amplas para gerenciar a incerteza (Thau et al., 2009). Segundo Van Den Bos e Lind (2002), uma impressão global sobre o que seria um tratamento justo baseado em procedimentos com informações justas, assim como a relação com os resultados, são propulsoras à gestão da incerteza. 
Diekmann, Barsness e Sondak (2004) afirmam que as percepções sobre as diversas dimensões da justiça, juntas ou separadas, podem gerar também um tratamento justo, contribuindo para a gestão da incerteza.

Analisando os avanços sobre justiça organizacional, de acordo com Santos (2010), pesquisadores identificaram que o interesse não parte exclusivamente do resultado e das recompensas. Embora nas dimensões da justiça organizacional não se tenha unanimidade na literatura, estas ocorrem apenas nas justiças distributiva e processual. Santos (2010) considera que há uma relação com a justiça processual, que reflete os mesmos resultados, sendo eles: processos de seleção, sistemas de avaliação de desempenho e procedimentos para aumentos salariais e promoções.

Para Assmar, Ferreira e Souto (2005, p. 445), a origem da justiça processual deve-se a "Thibaut e Walker (1975), que, influenciados pela tradição de pesquisa em procedimentos legais, desenvolveram um programa de investigação sistemática com o objetivo de comparar as reações das pessoas aos procedimentos de resolução de conflitos". Assmar, Ferreira e Souto (2005) salientam que as pessoas não se preocupam somente com a distribuição dos resultados, mas também com os critérios e os procedimentos adotados para efetivar esta distribuição, pois as pessoas tendem a aceitar resultados desfavoráveis quando percebem a presença de justiça atribuída no processo.

Depreende-se a importância tanto da distribuição de resultados de forma proporcional, quanto dos procedimentos e critérios adotados para a avaliação do desempenho. A justiça processual refere-se aos meios pelos quais os resultados são atribuídos, estabelecendo princípios que regem os indivíduos no processo decisório relativo à distribuição de recompensas ou promoção dos empregados nas organizações (Sudin, 2011).

Bakhshi, Kumar e Rani (2009) citam o estudo desenvolvido por Leventhal (1980), que apresenta uma esquematização dos fatores a serem considerados como mais justos nas organizações. Os achados da pesquisa indicaram os seguintes fatores considerados como mais justos: a consistência dos procedimentos ao longo do tempo, a ausência de vieses e interesses pessoais, a precisão das informações, os mecanismos para correção de falhas, atender aos princípios éticos e morais, e a possibilidade de corrigir decisões injustas.

Nesse sentido a pesquisa de Lind e Van Den Bos (2002) aborda a relação da incerteza com a justiça processual, e concluíram que as pessoas reagem mais fortemente à justiça em situações de alta incerteza do que em situações de baixa incerteza. Thau et al. (2009) confirmam essa lógica, visto que a alta incerteza ocasionada pela baixa transparência de informações da gestão agrava a relação de lealdade entre empregados e supervisores.

Diekmann, Barsness e Sondak (2004) constataram que a justiça processual é o melhor indicador para analisar a satisfação no trabalho, quando há insegurança sobre a forma de avaliação do desempenho utilizada pela empresa. As percepções da justiça processual são fundamentais para garantir o monitoramento dos resultados e sua precisão com os processos em que esses recursos são usados para corrigir as falhas nos resultados da organização (Sudin, 2011).

\subsection{PERCEPÇÃO DE JUSTIÇA NA AVALIAÇÃO DE DESEMPENHO}

A abordagem da avaliação de desempenho dos subordinados em uma organização está atrelada a sua motivação em melhorá-lo. A avaliação de desempenho está vinculada ao sistema de remunera- 
ção por recompensa, sendo que os subordinados atribuem especial importância para esta forma de avaliação (Lau, \& Buckland, 2001).

Considerando que a forma de avaliação é ditada por seus superiores, os subordinados podem atribuir determinada desconfiança aos métodos adotados, questionando segundo a sua percepção de justiça. Quanto maior for o número de medidas de desempenho, mais completa tende a ser a descrição da avaliação de desempenho (Moers, 2005).

No Quadro 1 são apresentadas algumas das definições identificadas na literatura no que tange a avaliação de desempenho.

Quadro 1: Conceitos de avaliação de desempenho

\begin{tabular}{|c|c|}
\hline Autores & Descrição \\
\hline Pontes (1996) & $\begin{array}{c}\text { A avaliação do desempenho é uma apreciação sistemática do } \\
\text { desempenho de cada pessoa no cargo e o seu potencial de } \\
\text { desenvolvimento futuro }\end{array}$ \\
\hline Carvalho e Nascimento (1997) & $\begin{array}{l}\text { A avaliação de desempenho constitui-se de uma série de técnicas com } \\
\text { a finalidade de obter informaçôes sobre o comportamento profissional } \\
\text { do avaliado durante o seu desempenho no trabalho. }\end{array}$ \\
\hline Moura (2000) & $\begin{array}{l}\text { O modo segundo o qual a empresa revê o desempenho dos seus } \\
\text { empregados através de métodos adequados. }\end{array}$ \\
\hline Fernandes e Caetano (2002) & $\begin{array}{l}\text { Um sistema formal e sistêmico que permite apreciar o trabalho } \\
\text { desenvolvido pelos empregados de uma organização. }\end{array}$ \\
\hline Lotta (2002) & $\begin{array}{l}\text { A avaliação de desempenho é um mecanismo que busca conhecer e } \\
\text { medir o desempenho dos indivíduos na organização, estabelecendo } \\
\text { uma comparação entre o desempenho esperado e o apresentado por } \\
\text { esses indivíduos. }\end{array}$ \\
\hline Sotomayor (2006) & $\begin{array}{l}\text { Avaliar o desempenho do trabalhador, em sentido estrito, consiste } \\
\text { em analisar os resultados do trabalho sem que, muitas vezes, o } \\
\text { trabalhador saiba o que dele é esperado. Integrar uma avaliaçăo de } \\
\text { desempenho ao sistema de gestão de desempenho implica que os } \\
\text { trabalhadores julguem os objetivos individuais que foram fixados e } \\
\text { recebam um feedback contínuo do seu desempenho e disponham } \\
\text { de informaçăăo sobre as medidas pelas quais o seu desempenho vai } \\
\text { ser avaliado. }\end{array}$ \\
\hline
\end{tabular}

Fonte: Adaptado de Santos (2010, p. 62)

Infere-se pelo exposto no Quadro 1, que a avaliação de desempenho implica avaliar o desempenho dos empregados para atingir determinado objetivo. Santos (2010) salienta que a avaliação de desempenho deve estar relacionada às estratégias da organização. Este processo objetiva a correção de falhas quando necessário. A avaliação de desempenho abrange a recompensa e a remuneração dos empregados, logo estes passam a se preocupar com a avaliação de seu desempenho (Lau, \& Sholihin, 2005).

As medidas de avaliação de desempenho podem ser classificadas de forma subjetiva ou pela sua diversidade. Moers (2005) caracteriza as avaliações de desempenho subjetivas como aquelas em que as medidas repassadas aos superiores não são claras, pois não existe um padrão para avaliação. A diversidade fornece aos superiores medidas baseadas em resultados. Dessa forma, o superior pode fazer escolhas de acordo com o seu julgamento.

Neste estudo focaliza-se a relação entre as medidas de avaliação de desempenho e a percepção de justiça processual. Assim, para a operacionalização da pesquisa consideraram-se as hipóteses e o instrumento de pesquisa desenvolvido no estudo de Hartmann e Slapnicar (2012), cuja síntese se apresenta na sequência.

\subsection{FORMULAÇÃO DAS HIPÓTESES DA PESQUISA}

Sob o aspecto da justiça processual, a presente pesquisa busca analisar os efeitos da incerteza e da tolerância a ambiguidade. Conforme Hartmann $(2000,2005)$ e Hartmann e Slapnicar (2012) a interli- 
gação desses efeitos com a justiça ainda é incipiente, sendo mais discutida na literatura de avaliação de desempenho.

Hartmann e Slapnicar (2012) conceituam-se a incerteza de tarefas como a variabilidade das tarefas executadas pelos gestores. Quando há alta incerteza de tarefas, presume-se maior dificuldade para reconhecer os esforços empreendidos e os resultados alcançados. Por outro lado, MacDonald (1970) salienta que a tolerância à ambiguidade remete à personalidade do indivíduo mediante informações incompletas e inconsistentes no ambiente de trabalho. Nesse caso, pessoas com baixa tolerância preferem informações mais precisas para executar uma atividade.

Com o intuito de verificar a relação entre as medidas de avaliação de desempenho e a justiça processual foram consideradas as variáveis formalidade, voz e medidas de resultado, levando em consideração o efeitos da incerteza das tarefas e da tolerância à ambiguidade.

\subsubsection{Formalidade}

Um aspecto a ser destacado no ambiente organizacional é a percepção da formalidade e seu efeito sobre a justiça processual na avaliação de desempenho. Conforme Moers (2005) a formalidade é definida como a objetividade do processo de avaliação de desempenho. Além desta pesquisa, a relação entre justiça processual e formalidade já foi identificada em Lau e Buckland (2001), Diekmann, Barsness e Sondak (2004), Hartmann (2005), Hartmann e Slapnicar (2009; 2012).

Em um processo de avaliação que ocorre de modo informal, a percepção de justiça tende a aumentar, devido a troca de informações de alta qualidade entre os superiores e seus subordinados (Hartmann, \& Slapnicar, 2012). Segundo esses autores, os superiores geralmente se utilizam de sistemas formais para o processo de avaliação de desempenho. Neste sentido, formula-se a seguinte hipótese:

H1: A relação entre a formalidade e a justiça processual é positiva para empregados com baixa incerteza de tarefas e com baixa tolerância à ambiguidade.

Em relação a incerteza de tarefas, Diekmann, Barsness e Sondak (2004) salientam que quando esta é alta, ocorre também uma elevada relação entre justiça e satisfação no trabalho. Hartmann e Slapnicar (2012) aduzem que a maior incerteza vincula-se ao menor julgamento da consistência das avaliações do desempenho. Porém, esses autores afirmam que em situações de maior incerteza, espera-se que o efeito positivo da formalidade seja anulado pelo efeito negativo da formalidade na avaliação da justiça processual em virtude da perda de relevância.

Em relação a tolerância à ambiguidade, quando os níveis de tolerância são menores, tem-se uma preferência por avaliações de desempenho mais diretas, com um feedback direto e específico (Hartmann, 2005). Nesse caso, apenas em ambiente com baixa tolerância à ambiguidade prevalece o efeito da formalidade na justiça processual.

\subsubsection{Voz}

Uma das variáveis estudadas nas percepções de justiça é a voz. Essa variável remete à prática de pessoas que são afetadas por informações relevantes, proferidas em um momento de decisão (Korsgaard, \& Roberson, 1995). Assmar, Ferreira e Souto (2005) salientam que Thibaut e Walker (1975) constataram que a voz é um dos procedimentos vistos como mais justos pelos empregados, por oferecerem a oportunidade de expressar suas opiniões. Assim, formulou-se a seguinte hipótese: 
H2: A relação entre voz e justiça processual é mais forte para empregados com alta incerteza de tarefas e com baixa tolerância à ambiguidade do que para empregados com baixa incerteza e alta tolerância à ambiguidade.

O efeito da voz na percepção de justiça equivale à precisão das informações no decorrer de algum processo. Envolve, de certa forma, a confiabilidade que se reflete na diminuição de incerteza, melhorando o desempenho do subordinado (Van Den Bos, \& Lind, 2002). Conforme Cremer e Sedikides (2005), o impacto da voz torna-se mais positivo quando se aumenta a incerteza. Os autores acrescentam ainda que a voz aumenta a percepção de controle, pois os gestores que possuem maiores níveis de incerteza nas tarefas, aumentam os controles. Em relação a tolerância à ambiguidade, Hartmann e Slapnicar (2012) expõem que os subordinados com menor tolerância compreendem uma situação com maior incerteza do que aqueles que possuem maior tolerância à ambiguidade.

\subsubsection{Medidas de resultado}

Considerando-se que a avaliação de desempenho está vinculada à motivação de atingir os objetivos propostos, a metodologia pela qual se avalia o esforço pode gerar desconfiança nos subordinados, questionando se é justa (Lau, \& Buckland, 2001). Neste sentido, formulou-se a seguinte hipótese:

H3: O uso de medidas de resultado tem um efeito negativo na percepção de justiça processual para os empregados com alta incerteza de tarefas e efeito positivo para empregados com baixa tolerância à ambiguidade.

O justo é avaliado pela representação fiel dos níveis de esforço, logo quanto maior o ambiente de incerteza, maior a demanda por uma avaliação de desempenho com base no esforço (Hartmann, 2005). O autor complementa que a avaliação do esforço deve levar em consideração as mudanças ao longo do tempo, influenciando na avaliação de desempenho. Portanto, os gestores com baixa tolerância à ambiguidade irão entender a importância da avaliação de desempenho pelo resultado, ao invés de somente pelo esforço

Hartmann e Slapnicar (2012) destacam que a utilização de medidas de esforço para a avaliação de desempenho é mais justa em ambientes de alta incerteza. E, para os empregados com menor tolerância à ambiguidade, a avaliação de desempenho pelo resultado é mais justa do que a medida pelo esforço.

\section{METODOLOGIA DA PESQUISA}

O delineamento metodológico desta pesquisa é do tipo descritivo quanto aos objetivos, de levantamento ou survey em se tratando dos procedimentos, e quantitativo quanto à abordagem do problema. A coleta dos dados foi realizada por meio da aplicação de um questionário aos empregados de uma cooperativa de produção. Utilizou-se o questionário desenvolvido por Hartmann e Slapnicar (2012), dividido em quatro blocos, fazendo inferências a três variáveis do estudo, o qual foi previamente testado pelos autores com cinco gerentes de três bancos diferentes, no intuito de avaliar a sua abrangência.

O instrumento de pesquisa foi aplicado aos empregados de uma cooperativa de produção da região Sul do Brasil. A cooperativa foi fundada em 1968, conta com 23 filiais e possui 325 empregados. Em 2011 atingiu um faturamento de 254 milhões. Tem suas atividades voltadas para a avicultura, suinocultura, leite, milho, feijão, soja, moinhos, fábricas de rações, atividades agro veterinárias, supermercados e postos de combustíveis. 
A seleção desta cooperativa de produção justifica-se por ser uma organização que adota uma política de remuneração baseada no desempenho dos seus empregados desde 2004, portanto demonstra já estar integrada em sua cultura. A forma diferenciada de gestão, por meio de um sistema cooperativo, é outro aspecto que motivou a escolha desta organização, podendo implicar diferenças nos resultados em relação ao estudo de base. Ainda que de forma secundária, foi considerada a acessibilidade ao quadro de pessoal da cooperativa, cujo acesso foi autorizado pela diretoria devido ao interesse nos resultados da pesquisa.

A população da pesquisa compreendeu os 325 empregados da cooperativa. A amostra da pesquisa abrangeu 119 empregados. A utilização da amostra diferenciada do estudo de Hartmann e Slapnicar (2012), que investigaram 178 gerentes de 12 bancos eslovenos, implicou atender o requisito de ser uma organização com a política de remuneração baseada no desempenho, permitindo, desta forma, observar as percepções de justiça junto aos empregados.

Para viabilizar a comparação dos resultados, adotaram-se os mesmos métodos estatísticos da pesquisa de base, desenvolvida por Hartmann e Slapnicar (2012). Primeiramente realizou-se a estatística descritiva, para a caracterização da amostra do estudo. Na sequência, foi efetuada uma análise fatorial para identificar os fatores relacionados à incerteza de tarefas e a tolerância à ambiguidade, com o uso do software SPSS (Statistical Package for the Social Science). Em seguida, os resultados foram classificados em duas amostras, uma que reflete um ambiente de baixa incerteza e outro de alta incerteza, 0 mesmo foi realizado com tolerância à ambiguidade.

A medição da formalidade (FORM) foi realizada a partir da percepção sobre a fixação de metas, as medidas de desempenho e a recompensa, realizada por uma escala Likert de cinco pontos. A variável medidas de resultado (OUT) teve por base a recompensa pautada no esforço. A variável voz (VOICE) foi examinada pela escala Likert de cinco pontos, da mesma forma como a variável justiça processual (PROCJ). A variável incerteza de tarefas (TU) foi avaliada com um instrumento de nove itens, elaborados por Withey, Daft e William (1983), e a tolerância à ambiguidade (TFA) com um instrumento de 13 itens, já abordados em um estudo de MacDonald (1970), ambos adotados por Hartmann e Slapnicar (2012).

Efetuadas as estimativas destas variáveis, calculou-se o Alfa de Cronbach, que é uma medida utilizada para verificar a confiabilidade de dois ou mais indicadores de um construto (Hair et al., 2005). Identificados estes fatores, aplicou-se o modelo de equações estruturais, utilizando-se o software SmartPLS versão 2.0. Conforme Hair et al. (2005), a modelagem de equações estruturais fornece a habilidade de ajustar diversas relações de dependência em somente um modelo.

\section{ANÁLISE E DESCRIÇÃO DOS DADOS}

\subsection{CARACTERIZAÇÃO DA AMOSTRA}

A população da pesquisa compreendeu os 325 empregados da cooperativa de produção objeto de estudo, já que todos recebem salário normativo mais participação nos resultados baseada no desempenho. A amostra da pesquisa consistiu dos 119 empregados desta cooperativa que responderam o instrumento de pesquisa. Os resultados mostram que $87,4 \%$ dos respondentes são do gênero masculino (104 respondentes). Do total dos respondentes, 47\% se encontram na faixa etária entre 21 a 30 anos, 26 \% entre 31 a 40 anos, 19\% entre 41 a 50 anos, 7\% na faixa etária até 20 anos e apenas um respondente 
se encontra na faixa etária acima de 50 anos. No estudo de Hartmann e Slapnicar (2012) a média de idade dos respondentes foi de 43,5 anos, com uma variação entre 29 e 58 anos.

A amostra incluiu empregados tanto do corpo administrativo, como da produção, vendas e área técnica. Quanto ao tempo de atuação na cooperativa identificou-se que 51\% (61 respondentes) estão na cooperativa a mais de 5 anos, o que mostra baixa rotatividade de pessoal. A maioria dos respondentes são empregados sem cargo de supervisão ou de coordenação, um total de 67\% (80 respondentes). Dos demais, 14\% (17 respondentes) têm cargo de supervisor, 12\% (14 respondentes) gerente de filial, $4 \%$ (5 respondentes) gerência corporativa, 2\% (2 respondentes) diretoria e 1 respondente tem cargo de presidência. Quanto a área de trabalho, 24\% (28 respondentes) trabalham na área administrativa da cooperativa, 37\% (44 respondentes) na produção, 17\% (20 respondentes) na área técnica, 23\% (27 respondentes) na área de vendas da cooperativa.

No que concerne a formação acadêmica, 19\% (23 respondentes) não possuem formação superior, $34 \%$ (40 respondentes) tem formação técnica, destacando-se a área agrícola, 39\% (46 respondentes) tem formação acadêmica em diversas áreas, com destaque em duas formações, Administração e Ciências Contábeis, que juntas somaram mais de 50\%, e $8 \%$ (10 respondentes) com especialização, sendo que não houve nenhum respondente com a titulação de mestre ou doutor na cooperativa em estudo.

\subsection{RESULTADOS DA PESQUISA}

Primeiramente efetuou-se uma análise fatorial para identificar os fatores relacionados a incerteza de tarefas e tolerância à ambiguidade, conforme apresentado na Tabela 1.

Tabela 1: Análise Fatorial

\begin{tabular}{|c|c|c|c|}
\hline \multicolumn{5}{|c|}{ Incerteza de Tarefas } \\
\hline Componentes & Total & \% de Variância & \% Acumulado \\
\hline Fator 1 & 3,070 & 34,113 & 34,113 \\
\hline Fator 2 & 1,614 & 17,935 & 52,047 \\
\hline Fator 3 & 1,156 & 12,846 & 64,893 \\
\hline \multicolumn{4}{|c|}{ Tolerância à Ambiguidade } \\
\hline Componentes & Total & \% de Variância & \% Acumulado \\
\hline Fator 1 & 2,273 & 17,484 & 37,484 \\
\hline Fator 2 & 1,755 & 13,497 & 43,981 \\
\hline Fator 3 & 1,691 & 13,007 & 53,438 \\
\hline Fator 4 & 1,228 & 9,449 & 61,976 \\
\hline Fator 5 & 1,110 & 8,538 & \\
\hline
\end{tabular}

Fonte: dados da pesquisa

A incerteza de tarefas foi medida por nove fatores, conforme apresentado no estudo de Withey, Daft e William (1983). Na análise fatorial foram identificados três fatores, conforme Tabela 1, que explicam juntos $64,89 \%$ da incerteza de tarefas. A metodologia utilizada para a determinação dos fatores foi o critério de Kaiser, que considera como fatores aqueles com valores superiores a 1,000. Para fins deste estudo utilizou-se apenas o primeiro fator encontrado, que individualmente explica $34,11 \%$ da incerteza de tarefas. Analisando este primeiro fator, percebe-se que apenas as questões 1, 5, 6, 7, 8 e 9, são as que explicam a incerteza de tarefas. Hartmann e Slapnicar (2012) encontraram na análise fatorial apenas 
2 fatores que explicam a incerteza de tarefas, considerando para análise apenas o primeiro fator. Pela metodologia de Kaiser, verificaram que as questões 1, 2, 3 e 4 do questionário de Withey, Daft e William (1983) explicaram a incerteza de tarefas.

Na sequência realizou-se a análise fatorial para a tolerância à ambiguidade. Esta medida foi avaliada por 13 fatores especificados no estudo de MacDonald (1970). Os resultados apontaram 5 fatores, que juntos explicam $61,98 \%$ da tolerância à ambiguidade. Para fins do estudo utilizou-se somente o primeiro fator, que individualmente explica 17,48\%. Desse modo, apenas as questões 1, 2, 3, 4 e 5 explicam a tolerância à ambiguidade. Hartmann e Slapnicar (2012) identificaram pela análise fatorial 3 fatores e que apenas as 7 primeiras questões de um total de 13 explicam a tolerância à ambiguidade.

Num segundo momento, a amostra do estudo foi dividida considerando os ambientes de baixa e alta incerteza e de baixa e alta tolerância à ambiguidade, tomando-se os scores da análise fatorial pela sua média. Em seguida, foi efetuado o Teste ANOVA, para averiguar se existem diferenças de médias nas variáveis do estudo. Os resultados são apresentados na Tabela 2.

Tabela 2: Resultados do Teste ANOVA

\begin{tabular}{|c|c|c|c|c|c|}
\hline & & \multicolumn{2}{|c|}{ Incerteza de Tarefas } & \multicolumn{2}{|c|}{ Tolerância à ambiguidade } \\
\hline & & Média & $\begin{array}{l}\text { Desvio } \\
\text { Padrão }\end{array}$ & Média & Desvio Padrão \\
\hline \multirow{2}{*}{ Formalidade } & Baixo & 3,28 & 2,45 & 2,93 & 2,32 \\
\hline & Alto & 3,48 & 2,15 & 3,64 & 2,23 \\
\hline \multirow{2}{*}{ Voz } & Baixo & $-0,98$ & 2,95 & $-0,58$ & 2,91 \\
\hline & Alto & 0,10 & 3,05 & $-0,28$ & 3,12 \\
\hline \multirow{2}{*}{ Medidas de resultado } & Baixo & 11,52 & 2,86 & 11,42 & 2,99 \\
\hline & Alto & 11,52 & 2,87 & 11,58 & 2,80 \\
\hline \multirow{2}{*}{ Justiça processual } & Baixo & 9,07 & 2,74 & 9,39 & 2,32 \\
\hline & Alto & 9,53 & 2,39 & 9,28 & 2,69 \\
\hline \multirow{2}{*}{ Incerteza } & Baixo & 1,11 & 0,67 & & \\
\hline & Alto & 3,49 & 0,63 & & \\
\hline \multirow{2}{*}{ Tolerância à ambiguidade } & Baixo & & & 0,64 & 0,43 \\
\hline & Alto & & & 2,02 & 0,45 \\
\hline
\end{tabular}

Fonte: dados da pesquisa

Nos resultados do Teste Anova apresentados na Tabela 2, nota-se que há diferenças de médias apenas entre as variáveis incerteza de tarefas e tolerância à ambiguidade, quando relacionadas entre si, não havendo, dessa forma, maiores problemas para o estudo. Os resultados são semelhantes aos encontrados por Hartmann e Slapnicar (2012), exceto na variável medidas de resultado.

Em seguida efetuou-se uma regressão para analisar a relação entre incerteza de tarefas e tolerância à ambiguidade, conforme apresentado na Tabela 3.

Tabela 3: Resultados da Regressão

\begin{tabular}{|l|c|c|c|c|c|}
\hline & & \multicolumn{2}{|c|}{ Incerteza de Tarefas } & \multicolumn{2}{|c|}{ Tolerância à ambiguidade } \\
\hline \multirow{3}{*}{ Incerteza } & Pearson Correlation & 1 &, $246^{* *}$ & 2,93 & 2,32 \\
\cline { 2 - 6 } & Sig. (2-tailed) & &, 007 & 3,64 & 2,23 \\
\cline { 2 - 6 } & $\mathrm{N}$ & 119 & 119 & & \\
\hline \multirow{3}{*}{ Tolerância } & Pearson Correlation &, $246^{* *}$ & 1 & $-0,58$ & 2,91 \\
\cline { 2 - 7 } & Sig. (2-tailed) &, 007 & & & \\
\cline { 2 - 6 } & $\mathrm{N}$ & 119 & 119 & $-0,28$ & 3,12 \\
\hline
\end{tabular}

**. Correlation is significant at the 0.01 level ( 2 -tailed) Fonte: dados da pesquisa. 
Conforme os resultados da Tabela 3, há uma correlação significante entre as variáveis, porem estas são consideradas fracas. Dessa maneira, não afetará os resultados da pesquisa. No estudo de Hartmann e Slapnicar (2012) a correlação entre incerteza de tarefas e tolerância à ambiguidade não foi significante.

Num último momento da análise, aplicou-se o modelo de equações estruturais, com a finalidade de confirmar ou refutar as hipóteses de pesquisa. Nesse sentido, analisou-se a confiabilidade do modelo de mensuração, conforme apresentado na Tabela 4.

Tabela 4: Critérios de Qualidade

\begin{tabular}{|c|c|c|c|c|c|}
\hline & AVE & $\begin{array}{c}\text { Composite } \\
\text { Reliability }\end{array}$ & R2 & $\begin{array}{c}\text { Cronbach's } \\
\text { Alpha }\end{array}$ & Redundancy (Q2) \\
\hline Esforço & 0,6133 & 0,8559 & 0,0000 & 0,8087 & 0,0000 \\
\hline Formalidade & 0,3495 & 0,0000 & 0,0000 & 0,2767 & 0,0000 \\
\hline Voz & 0,4021 & 0,0550 & 0,0000 & 0,0903 & 0,0000 \\
\hline Justiça Proc. & 0,7685 & 0,9071 & 0,3445 & 0,8558 & 0,1077 \\
\hline
\end{tabular}

Fonte: dados da pesquisa

Observa-se na Tabela 4 que a validade discriminante do construto é representada pela AVE (variância média) e pelas cargas do modelo. Para a AVE indicar confiabilidade, ela deve ser superior a 0,5, ou seja, a variável é capaz de explicar 50\% da variância dos indicadores.

Com base nos achados, apenas 2 medidas (esforço e justiça processual) atendem este requisito, assim como para os requisitos de confiabilidade composta (os fatores formalidade e voz, são pouco explicativos) e do Alfa de Cronbach (valor superior a 0,7). Diante disso, optou-se pela permanência das demais variáveis, assegurando o constructo proposto por Hartmann e Slapnicar (2012).

A validade preditiva é confirmada pelo índice de Stone-Geisser (Q2), apresentando valores superiores a 0 , o que vem ao encontro do estudo base. Na Tabela 5, apresentam-se os valores encontrados para a validade discriminante do modelo.

Tabela 5: Cross loadings

\begin{tabular}{|c|c|c|c|c|}
\hline & Esforço & Formalidade & Voz & Justiça Processual \\
\hline ESF_1 & 0,6845 & $-0,3507$ & $-0,5178$ & 0,2415 \\
\hline ESF_2 & 0,4667 & $-0,1082$ & $-0,0558$ & 0,0337 \\
\hline ESF_3 & 0,9418 & $-0,3482$ & $-0,3794$ & 0,422 \\
\hline ESF_4 & 0,9379 & $-0,3549$ & $-0,379$ & 0,4733 \\
\hline FORM_1 & $-0,3413$ & 0,7529 & 0,363 & $-0,3235$ \\
\hline FORM_2 & 0,2497 & $-0,5929$ & $-0,397$ & 0,0823 \\
\hline FORM_3 & $-0,1686$ & 0,733 & 0,2976 & $-0,3376$ \\
\hline FORM_4 & 0,1798 & $-0,6268$ & $-0,3117$ & 0,1327 \\
\hline FORM_5 & 0,3518 & 0,1837 & $-0,1589$ & $-0,0738$ \\
\hline FORM_6 & 0,4576 & $-0,4632$ & $-0,3172$ & 0,2376 \\
\hline JUST_1 & 0,0665 & $-0,2214$ & $-0,2388$ & 0,6946 \\
\hline JUST_2 & 0,4394 & $-0,4029$ & $-0,5028$ & 0,9546 \\
\hline JUST_3 & 0,5123 & $-0,4003$ & $-0,5307$ & 0,9548 \\
\hline VOZ_1 & $-0,0941$ & 0,3001 & 0,6229 & $-0,2639$ \\
\hline VOZ_2 & $-0,0547$ & $-0,0687$ & $-0,214$ & 0,013 \\
\hline VOZ_3 & $-0,4775$ & 0,3661 & 0,6771 & $-0,3612$ \\
\hline VOZ_4 & 0,3654 & $-0,3808$ & $-0,7348$ & 0,3301 \\
\hline VOZ_5 & $-0,4023$ & 0,3603 & 0,7509 & $-0,2757$ \\
\hline VOZ_6 & 0,2189 & $-0,2489$ & $-0,6452$ & 0,4654 \\
\hline & & & & \\
\hline
\end{tabular}


A validade discriminante do modelo foi determinada pelo cross loading, conforme Tabela 5. O loading de cada indicador é superior a todos os cross loadings. Por exemplo, percebe-se que a questão 1 possui a maior carga na variável que remete ao esforço, e assim sucessivamente. Estes resultados coadunam com os de Hartmann e Slapnicar (2012).

Diante do exposto, efetuou-se a análise pelo modelo de equações estruturais em ambientes de baixa e alta incerteza de tarefas e em ambientes de baixa e alta tolerância à ambiguidade. Os resultados são apresentados nas Tabelas 6 e 7. Na Tabela 6 apresentam-se os resultados relativos a incerteza de tarefas.

Tabela 6: Modelo estrutural para as duas amostras de Incerteza de Tarefas

\begin{tabular}{|c|c|c|c|c|c|c|c|}
\hline & \multicolumn{3}{|c|}{ Baixa Incerteza de Tarefas } & \multicolumn{3}{|c|}{ Alta Incerteza de tarefas } & \multirow{2}{*}{$\begin{array}{l}\text { Diferença de } \\
\text { caminhos }\end{array}$} \\
\hline & Amostra Original & Média & Standard Erro & Amostra Original & Média & Standard Erro & \\
\hline $\begin{array}{c}\text { Esforço-> } \\
\text { Justiça }\end{array}$ & 0,2370 & 0,2462 & 0,1136 & 0,1962 & 0,2141 & 0,1057 & 0,0408 \\
\hline $\begin{array}{c}\text { Formalid.-> } \\
\text { Justiça }\end{array}$ & $-0,1548$ & $\stackrel{-}{0,1560}$ & 0,1680 & 0,3398 & 0,3508 & 0,1106 & 0,4946 \\
\hline Voz-> Justiça & $-0,3363$ & $\overline{0}, \overline{-}$ & 0,3520 & 0,1784 & 0,2088 & 0,0975 & 0,5147 \\
\hline
\end{tabular}

Fonte: dados da pesquisa.

Observa-se na Tabela 6 o comportamento das variáveis em ambientes de baixa e de alta incerteza de tarefas. Em se tratando da avaliação de desempenho, que está vinculada a motivação de atingir os objetivos propostos pela organização, avalia-se o esforço efetuado pelo subordinado para tal. Nesse sentido, percebe-se que existe uma pequena diferença entre as duas amostras, resultado este encontrado também por Hartmann e Slapnicar (2012).

Na relação da formalidade com a justiça processual na avaliação de desempenho, encontrou-se uma diferença grande entre as duas amostras. A relação foi superior em ambientes de alta incerteza de tarefas, ao contrário do estudo de Hartmann e Slapnicar (2012), que encontrou maior evidencia da formalidade em ambientes de baixa incerteza de tarefas.

Cremer e Sedikides (2004), Diekmann, Barsness e Sondak (2004) corroboram que em ambientes de alta incerteza de tarefas a relação entre a justiça e a satisfação do trabalho tende a aumentar. No que concerne aos procedimentos de avaliação de desempenho, Hartmann (2005), Lau e Moser (2008) afirmam que, em ambientes caracterizados como de elevada incerteza, o nível de controle das tarefas é menor. Conforme Hartmann e Slapnicar (2012), a maior incerteza de tarefas está associada a menor consistência no julgamento do desempenho dos subordinados, de maneira que eles consideram a sua avaliação injusta.

Outra variável analisada nas percepções de justiça é a voz. Nota-se que a, maior diferença foi encontrada nas duas amostras para a incerteza de tarefas. Constatou-se maior relação da voz com justiça processual em ambientes de baixa incerteza. Corroboram-se assim os achados do estudo de Hartmann e Slapnicar (2012), que também encontraram as maiores diferenças na variável voz.

Na Tabela 7 são apresentados os resultados relativos a tolerância à ambiguidade nas duas amostras (baixa e alta).

Tabela 7: Modelo estrutural para as duas amostras de Tolerância à Ambiguidade

\begin{tabular}{|c|c|c|c|c|c|c|c|}
\hline & \multicolumn{3}{|c|}{ Baixa Tolerância à Ambiguidade } & \multicolumn{3}{c|}{ Alta Tolerância à Ambiguidade } & \multirow{2}{*}{$\begin{array}{c}\text { Diferença de } \\
\text { caminhos }\end{array}$} \\
\cline { 2 - 9 } & Amostra Original & Média & Standard Erro & Amostra Original & Média & Standard Erro & $-0,1016$ \\
\hline Esforço-> Justiça & 0,1893 & 0,1889 & 0,0882 & 0,2909 & 0,2975 & 0,0914 & 0,1600 \\
\hline Formalidade-> Justiça & 0,4483 & 0,4484 & 0,0957 & 0,0699 & 0,0321 & 0,3784 \\
\hline Voz-> Justiça & 0,1998 & 0,1852 & 0,1692 & 0,3443 & 0,3580 & 0,1112 & $-0,1445$ \\
\hline
\end{tabular}


Em relação a variável esforço (que caracteriza as medidas de resultado), verifica-se na Tabela 7 uma pequena diferença entre as amostras, sendo maior a relação desta variável com justiça processual em ambientes de alta tolerância à ambiguidade. Este resultado difere da pesquisa de Hartmann e Slapnicar (2012), que encontraram a maior diferença para esta variável, quando comparada com as demais, apresentando maior relação em ambientes de baixa tolerância à ambiguidade. A maior relação da variável esforço em ambientes de alta tolerância à ambiguidade é justificada por Hartmann (2005), por se tratar de uma característica que permeia situações de indiferença para os subordinados quanto a avaliação do desempenho por esforço ou por medidas quantitativas de resultado.

Por sua vez, a formalidade apresentou maior diferença de caminhos quando comparada com as demais relações efetuadas, com predominância em ambientes de baixa tolerância à ambiguidade. Hartmann e Slapnicar (2012) encontraram apenas uma pequena diferença para alta tolerância à ambiguidade. A predominância das relações entre formalidade e justiça processual em ambientes de baixa incerteza indica maior preferência dos subordinados em obter um feedback da sua avaliação de desempenho.

A relação da justiça processual com voz dos subordinados apresentou-se mais forte em ambientes com alta tolerância à ambiguidade, corroborando com os resultados encontrados por Hartmann e Slapnicar (2012).

\subsection{DISCUSSÃO DAS HIPÓTESES A PESQUISA}

Na Figura 1, apresenta-se o desenho estrutural do modelo proposto para, na sequência, confirmar ou refutar as hipóteses de pesquisa.

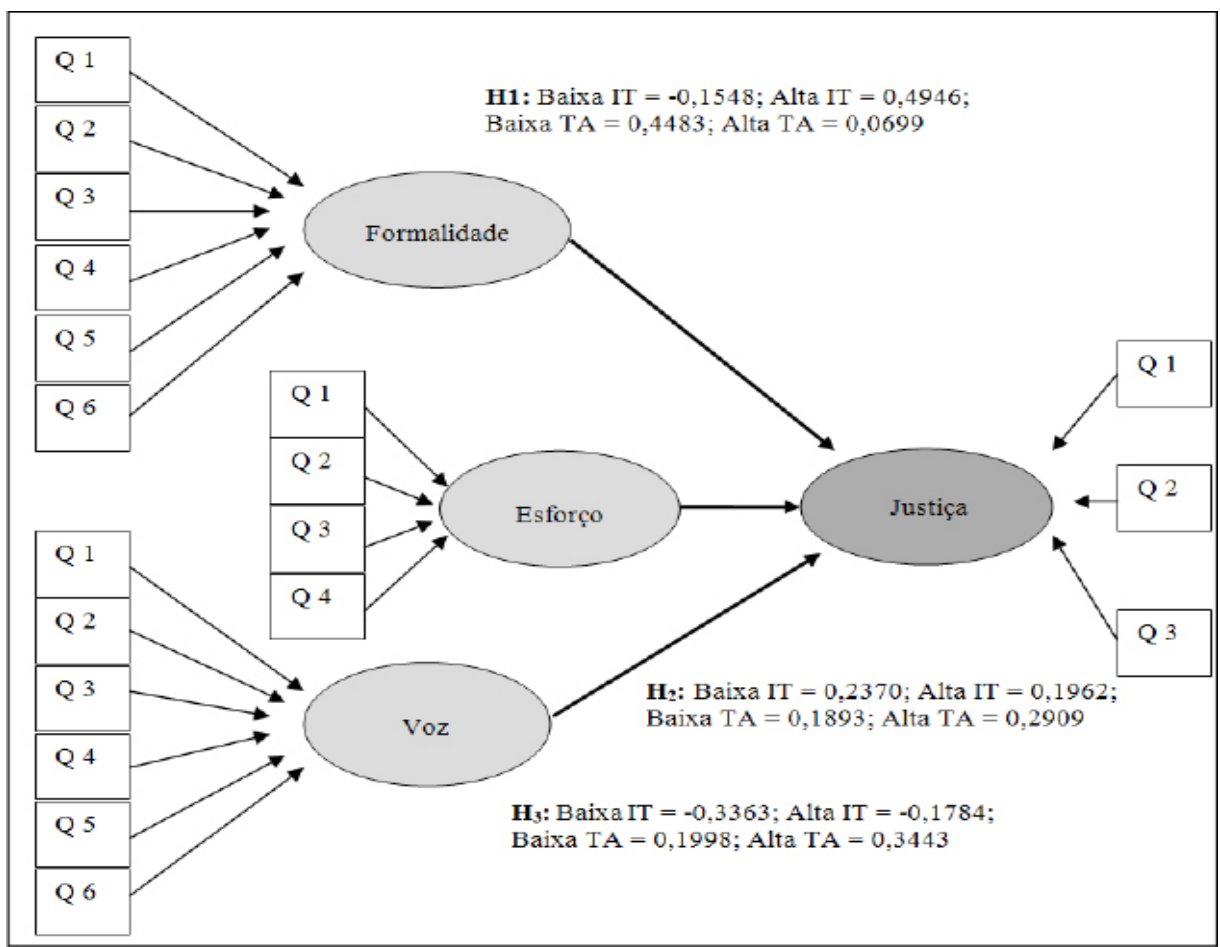

Figura 1: Modelo estrutural da pesquisa

Fonte: dados da pesquisa. 
Observa-se na Figura 1 que a hipótese H1 propõe que a relação entre a formalidade e a justiça processual é positiva para empregados com baixa incerteza de tarefas e com baixa tolerância à ambiguidade. No entanto, os resultados da pesquisa apontam que ocorre uma relação positiva entre formalidade e justiça processual, sendo maior em ambientes de alta incerteza de tarefas (com uma diferença de caminhos de 0,4946) e de baixa tolerância à ambiguidade (com uma diferença de caminhos de 0,3784).

Fazendo-se um comparativo com o estudo de Hartmann e Slapnicar (2012), o resultado encontrado pelos autores é contrário ao presente estudo. Os autores constataram que a relação entre formalidade e justiça é maior em ambientes de baixa incerteza de tarefas e alta tolerância à ambiguidade.

Em relação a incerteza de tarefas, Diekmann, Barsness e Sondak (2004) corroboram que em ambientes de alta incerteza a relação entre a justiça e a satisfação do trabalho aumenta. A maior incerteza de tarefas é associada com uma menor possibilidade de auxiliar na avaliação. Em relação a ambiguidade, Hartmann e Slapnicar (2012) aduzem que a amostra pertencente a menor tolerância à ambiguidade tem maior preferência para obter um feedback da avaliação de desempenho.

Diante dos resultados, confirma-se parcialmente a hipótese $\mathrm{H} 1$, quando se assume que existe uma relação positiva entre formalidade e justiça em ambientes de baixa tolerância à ambiguidade. Em seguida, analisou-se a segunda hipótese $(\mathrm{H} 2)$ de pesquisa, relativa a voz dos empregados. Nesta hipótese assume-se que a relação entre voz e justiça processual é mais forte para empregados com alta incerteza de tarefas e com baixa tolerância à ambiguidade do que para empregados com baixa incerteza de tarefas e alta tolerância à ambiguidade.

Conforme os resultados da pesquisa, essa relação é explicada em ambientes de baixa incerteza (com uma diferença de caminhos de 0,5147) e ambientes de alta tolerância à ambiguidade (com diferença de caminhos de -0,1445). Os achados de Hartmann e Slapnicar (2012) reportam a explicação desta relação em ambientes de alta incerteza de tarefas e alta tolerância à ambiguidade. Dessa forma, a H2 é rejeitada.

Em relação ao esforço dos empregados, a hipótese de pesquisa $\mathrm{H} 3$ assume que o uso de medidas de resultado tem um efeito negativo na percepção de justiça processual para os empregados com alta incerteza de tarefas e efeito positivo para empregados com baixa tolerância à ambiguidade. Os resultados apontam que o efeito negativo sobre a percepção de justiça em relação ao uso das medidas de resultado (incerteza de tarefas) não se confirma, resultado este encontrado também por Hartmann e Slapnicar (2012). Outro achado da pesquisa coincide com a relação entre as variáveis apontadas em ambientes de baixa incerteza e de alta tolerância à ambiguidade.

Nesse sentido a hipótese de pesquisa $\mathrm{H} 3$, relacionada as medidas de resultado, não se confirma. De acordo com Hartmann e Slapnicar (2012), os empregados pertencentes a amostra de baixa tolerância à ambiguidade irão entender a importância de avaliar o desempenho não somente pelo esforço mas sim pelo resultado, o que não se confirma no presente estudo.

No Quadro 2, apresenta-se um comparativo dos resultados das hipóteses desta pesquisa com o estudo de Hartmann e Slapnicar (2012).

Quadro 2: Comparação dos resultados de Hartmann e Slapnicar (2012) e desta pesquisa

\begin{tabular}{|c|c|c|c|}
\hline \multirow{2}{*}{ Variável } & Hipóteses & $\begin{array}{c}\text { Resultado de Hartmann e } \\
\text { Slapnicar (2012) }\end{array}$ & $\begin{array}{c}\text { Resultado da presente } \\
\text { pesquisa }\end{array}$ \\
\hline \multirow{2}{*}{ Formalidade } & $\begin{array}{c}\text { H1a: A relação entre a formalidade e a justiça } \\
\text { processual é positiva para os empregados com baixa } \\
\text { incerteza de tarefas. }\end{array}$ & Hipótese confirmada & Hipótese não confirmada \\
\cline { 2 - 5 } & $\begin{array}{c}\text { H1b: A relação entre a formalidade e a justiça } \\
\text { processual é positiva para os empregados com baixa } \\
\text { tolerância à ambiguidade. }\end{array}$ & Hipótese não confirmada & Hipótese confirmada \\
\hline
\end{tabular}




\begin{tabular}{|c|c|c|c|}
\hline \multirow{2}{*}{ Voz } & $\begin{array}{c}\text { H2a: A relação positiva entre a voz e justiça processual } \\
\text { é mais forte para os empregados com alta incerteza } \\
\text { de tarefas. }\end{array}$ & Hipótese confirmada & Hipótese não confirmada \\
\cline { 2 - 5 } & $\begin{array}{c}\text { H2b: A relação entre a voz e a justiça processual é } \\
\text { mais forte para os empregados com baixa tolerância à } \\
\text { ambiguidade. }\end{array}$ & Hipótese não confirmada & Hipótese não confirmada \\
\hline \multirow{2}{*}{ Medidas de } & $\begin{array}{c}\text { H3a: O uso de medidas de resultado tem um efeito } \\
\text { negativo na percepção de justiça processual para os } \\
\text { empregados com alta incerteza de tarefas. }\end{array}$ & Hipótese não confirmada & Hipótese não confirmada \\
\cline { 2 - 5 } Resultado & $\begin{array}{c}\text { H3b: O uso de medidas de resultado tem um efeito } \\
\text { positivo para empregados com baixa tolerância à } \\
\text { ambiguidade. }\end{array}$ & Hipótese confirmada & Hipótese não confirmada \\
\hline
\end{tabular}

Fonte: dados da pesquisa.

No Quadro 2, é possível observar que os resultados de Hartmann e Slapnicar (2012) evidenciam que a percepção de justiça nas medidas de resultado, diversidade destas medidas e voz são dependentes do grau de incerteza de tarefas que os gerentes executam. Observa-se ainda que a percepção de justiça nas medidas de desempenho é condicionada a tolerância à ambiguidade.

Na presente pesquisa verificou-se que a percepção de justiça advinda da formalidade do processo de avaliação de desempenho é dependente do grau de incerteza das tarefas que os empregados executam. As demais relações (voz e medidas de resultado) não foram comprovadas de forma plena, seja quanto à incerteza de tarefas e em relação a tolerância à ambiguidade.

Especula-se que a diferenças encontradas podem decorrer da amostra utilizada. A pesquisa de Hartmann e Slapnicar (2012) foi desenvolvida com 178 gestores de 12 bancos eslovenos, enquanto que a presente pesquisa foi desenvolvida com 119 empregados de uma cooperativa de produção brasileira. Todavia, possíveis determinantes para as diferenças entre ambas as pesquisas, como várias instituições financeiras e um caso único de cooperativa, setores econômicos divergentes e aspectos culturais de países, instigam mais pesquisas e se apresentam como uma lacuna para novos estudos.

Em síntese, os resultados empíricos desta pesquisa, juntamente com a discussão teórico-empírica realizada por Hartmann e Slapnicar (2012), levam ao consenso acerca da influência da incerteza de tarefas sobre a formalidade do processo de avaliação de desempenho. Relação esta também identificada nas pesquisas de Lau e Buckland (2001), Diekmann, Barsness e Sondak (2004), Hartmann (2005). Por outro lado, instigam a realização de mais pesquisas sobre as relações da justiça processual com a voz e as medidas de resultado.

\section{CONSIDERAÇÕES FINAIS}

O estudo verificou a relação entre as medidas de avaliação de desempenho e a percepção de justiça processual à luz da incerteza e da tolerância à ambiguidade. Pesquisa descritiva com abordagem quantitativa foi realizada por meio de um levantamento junto a 119 empregados de uma cooperativa de produção da região Sul do Brasil. Aplicou-se um questionário desenvolvido no estudo de Hartmann e Slapnicar (2012), composto pelas variáveis formalidade, voz e medidas de resultado que influenciam na avaliação de desempenho e nas percepções de justiça processual. O estudo de Hartmann e Slapnicar (2012) foi desenvolvido em 12 bancos eslovenos, com uma amostra equivalente a 178 gerentes que responderam o questionário.

Os respondentes desta pesquisa são, em sua maioria, do gênero masculino (104 respondentes de um total de 119), com uma faixa etária entre 21 a 30 anos (47 respondentes). Em relação ao tempo de atuação na cooperativa, $51 \%$ dos empregados estão na cooperativa a mais de 5 anos e $67 \%$ não ocupam cargo de supervisão ou coordenação. Quanto a área em que trabalham, 24\% estão em fun- 
ções administrativas, 37\% em funções de produção, 17\% na área técnica e 23\% na área de vendas da cooperativa. Na formação dos respondentes, destaca-se que 34\% possuem alguma formação técnica e $39 \%$ possuem formação acadêmica.

Quanto à análise das hipóteses, na primeira buscou-se analisar a relação entre formalidade e justiça processual. Os resultados indicaram uma relação mais forte quando os empregados se encontram em ambientes de alta incerteza de tarefas e baixa tolerância à ambiguidade. A hipótese $\mathrm{H} 1$ é aceita parcialmente, uma vez que a relação para ambientes de baixa incerteza não foi encontrada. Hartmann e Slapnicar (2012) constataram em seu estudo relação em ambientes de baixa incerteza e de alta tolerância à ambiguidade. $O$ resultado que remete a alta incerteza de tarefas é justificado por Cremer e Sedikides (2004), Diekmann, Barsness e Sondak (2004) de maneira que a relação entre a justiça e a satisfação do trabalho tende a aumentar. Hartmann (2005), Lau e Moser (2008) acrescentam que esse ambiente de alta incerteza reflete um menor controle das tarefas desempenhadas pelos subordinados.

Para a segunda hipótese, que analisou a relação da voz com a percepção de justiça, se esperava uma relação mais forte em ambientes de alta incerteza e de baixa tolerância à ambiguidade. Os resultados indicaram uma situação contrária ao esperado, implicando na rejeição da hipótese H2. Os resultados da pesquisa de Hartmann e Slapnicar (2012) indicaram relação entre voz e justiça processual em ambientes de alta incerteza e de alta tolerância à ambiguidade. Thibaut e Walker (1975), Assmar, Ferreira e Souto (2005) observaram que voz tem relação direta com justiça processual, pois dando voz aos subordinados na avaliação de desempenho, tem-se um efeito positivo na sua percepção de justiça processual.

Na relação das medidas de resultado com a percepção de justiça processual, que compreende a terceira hipótese da pesquisa, esperava-se uma relação negativa para alta incerteza de tarefas e um efeito positivo para alta tolerância à ambiguidade. Os resultados evidenciaram uma relação contrária a esperada, implicando em uma relação positiva entre as variáveis em ambientes de baixa incerteza e de alta tolerância à ambiguidade. Diante disso rejeita-se a hipótese H3. Os resultados do estudo de Hartmann e Slapnicar (2012) apontaram uma relação entre voz e percepção de justiça em ambientes com baixa incerteza de tarefas e baixa tolerância à ambiguidade. Os autores inferiram que os empregados pertencentes a ambientes de alta tolerância à ambiguidade podem não compreender a importância de avaliar o desempenho somente pelo esforço, mas sim pelo resultado.

Conclui-se que as três medidas analisadas, formalidade, voz e medidas de resultado, afetam a percepção de justiça processual dos empregados. No entanto, constataram-se diferenças entre as amostras relativas a incerteza de tarefas e a tolerância à ambiguidade. Diante das limitações deste estudo, recomenda-se para estudos futuros ampliar a amostra de empresas, além de considerar outra amostra de respondentes (gerentes) para analisar o comportamento das variáveis. Sugere-se ainda o emprego de outras medidas que possam influenciar na percepção de justiça processual de empregados ou gestores.

\section{REFERÊNCIAS}

Assmar, E. M. L., Ferreira, M. C., \& Souto, S. O. (2005). Justiça organizacional: uma revisão crítica da literatura. Psicologia: Reflexão e Crítica, 18(3), 443-453.

Bakhshi, A., Kumar, K., \& Rani, E. (2009). Organizational justice perceptions as predictor of job satisfaction and organization commitment. International Journal of Business and Management, 4(9), 145-154. 
Campos, G. R., \& Gozer, I. C. (2008). Gestão estratégica de custos nas cooperativas agropecuárias do estado do Paraná: nível de intensidade no uso das informações de custos para tomada de decisão e controle. Revista de Ciências Empresariais, 9(1/2), 155-172.

Carvalho, A. V., \& Nascimento, L. P. (1997). Administração de recursos humanos. 2 ed. São Paulo: Pioneira.

Cremer, D., \& Sedikides, C. (2005). Self-uncertainty and responsiveness to procedural justice. Journal of Experimental Social Psychology, 45(1), 157-173.

Diekmann, K. A., Barsness, Z. I., \& Sondak, H. (2004). Uncertainty, fairness perceptions and job satisfaction: a field study. Social Justice Research, 17(3), 237-255.

Fernandes, A., \& Caetano, A. (2002). A avaliação do desempenho. In: Caetano, A., \& Vala, J. (Org.). Gestão de recursos humanos: contextos, processos e técnicas. 2. ed. Lisboa: Editora RH. pp. 357-387.

Hair, J. F., Anderson, R. E., Tatham, R. L., \& Black, W. C. (2005). Análise multivariada de dados. 5. ed. Porto Alegre: Bookman.

Hartmann, F. (2005). The effects of tolerance for ambiguity and uncertainty on the appropriateness of accounting performance measures. Journal of Accounting, Finance and Business Studies, 41(3), 241-264.

Hartmann, F. (2000). The appropriateness of RAPM: toward the further development of theory. Accounting, Organizations and Society, 25(4-5), 451-482.

Hartmann, F., \& Slapnicar, S. (2009). How formal performance evaluation affects trust between superior and subordinate managers. Accounting, Organizations and Society, 34(6-7), 722-737.

Hartmann, F., \& Slapnicar, S. (2012). The perceived fairness of performance evaluation: the role of uncertainty. Management Accounting Research, 23(1), 17-33.

Korsgaard, A. M., \& Roberson, L. (1995). Procedural justice in performance evaluation: the role of instrumental and non-instrumental voice in performance appraisal discussion. Journal of Management, 21(4), 657-669.

Lau, C. M., \& Buckland, C. (2001). Budgeting-role of trust and participation: a research note. Journal of Accounting, Finance and Business Studies, 37(3), 369-388.

Lau, C. M., \& Moser, A. (2008). Behavioral effects of nonfinancial performance measures: the role of procedural fairness. Behavioral Research in Accounting, 20(2), 55-71.

Lau, C. M., \& Sholihin, M. (2005). Financial and nonfinancial performance measure: how do they affect job satisfaction? The British Accounting Review, 37(4), 389-413.

Leventhal, G. S. (1980). What should be done with equity theory? New approaches to the study of fairness in social relationships. In: Gergen, K. J., Greenberg, M. S., \& Willis, R. H. Social exchange: advances in theory and research. New York: Plenum. pp. 27-55.

Lind, E. A., \& Van Den Bos, K. (2002). When fairness works: toward a general theory of uncertainty management. Research in Organizational Behaviour, 24(1), 181-223.

MaCdonald, A. P. (1970). Revised scale for ambiguity tolerance: reliability and validity. Psychological Reports, 26(6), 791-798.

Moers, F. (2005). Discretion and bias in performance evaluation: the impact of diversity and subjectivity. Accounting, Organizations and Society, 30(1), 67-80.

Moura, E. (2000). Gestão de recursos humanos: influências e determinantes do desempenho. Lisboa: Edições Silabo. 
Pontes, B. R. (1996). Avaliação de desempenho: nova abordagem. 6. ed. São Paulo: LTR.

Santos, V. (2010). Percepção de justiça na avaliação de desempenho versus folga organizacional dos controllers: um estudo em empresas com sistemas de remuneração por recompensa. Dissertação de Mestrado, Universidade Regional de Blumenau, Blumenau, SC, Brasil.

Sudin, S. (2011). Fairness of and satisfaction with performance appraisal process. Journal of Global Management, 2(1), 66-83.

Thau, S., Bennett, R. J., Mitchell, M. S., \& Marrs, M. B. (2009). How management style moderates the relationship between abusive supervision and workplace deviance: an uncertainty management theory perspective. Organizational Behavior and Human Decision Processes, 108(1), 79-92.

Thibaut, J., \& Walker, L. (1975). Procedural justice: a psychological analysis. Hillsdale, NJ: Erlbaum.

Van Den Bos, K., \& Lind, E. A. (2002). Uncertainty management by means of fairness judgments. In: Zanna, M. P. (ed.). Advances in experimental social psychology. Vol. 34. San Diego: Academic Press. pp. 1-60.

Withey, M., Daft, R. L., \& William, H. C. (1983). Measures of Perrow's work unit technology: an empirical assessment and a new scale. Academy of Management Journal, 26(1), 45-63. 